\title{
Benefits of Enterprise Integration: Review, Classification, and Suggestions for Future Research
}

\author{
Ariyan Fazlollahi ${ }^{1}$, Ulrik Franke ${ }^{1,2}$, and Johan Ullberg ${ }^{1}$ \\ ${ }^{1}$ Industrial Information and Control Systems, KTH Royal Institute of Technology, \\ Osquldas v. 12, SE-10044 Stockholm, Sweden \\ ariyan@kth.se, \{ulrikf, johanu\}@ics.kth.se \\ ${ }^{2}$ Swedish Defence Research Agency, SE-164 90 Stockholm, Sweden
}

\begin{abstract}
This article reports the findings of a literature review concerning the potential benefits of Enterprise Integration (EI) for organizations. The review reveals the current state of the scientific literature concerning the potential benefits of EI, classified using a conceptual model of the enterprise. We believe that the results provide a consolidated and comprehensive picture of such potential benefits, useful as a baseline for future research. Additionally, the review is expected to assist practitioners in establishing business cases for EI by means of scientifically grounded reasoning about how EI benefits can contribute to the achievement of certain business goals. Additionally, results could be employed to develop methods or models capable of measuring such benefits in financial terms.
\end{abstract}

Keywords: Enterprise Integration, Enterprise Systems, Benefits, Classification, Literature Review.

\section{Introduction}

Large multinational enterprises operating in global markets, continuous development of computing technologies, and the extended, dispersed and continuously interconnected enterprise information systems, has caused a fundamental transformation of the economy [1]. Moreover, the migration of organizations toward greater complexity with global presence, sever economic pressures, the need for enhanced innovation and more participative and learning-oriented management practices call for change in organizational life regarding the technology-organizational relationship [2]. Considering such forces, studies in organizational sciences generally agree on the evolution of organizational forms towards decentralized and more flexible approaches and structures [2].

In order to evolve, survive and thrive in these competitive markets, enterprises must find a way to cope with rapid changes in both internal and external environments. As a strategy to be able to rapidly respond to a changing environment, business functions can be integrated in order to efficiently utilize information technology, and share data with third-party actors in the marketplace [3].

The original version of this chapter was revised: The copyright line was incorrect. This has been corrected. The Erratum to this chapter is available at DOI: 10.1007/978-3-642-33068-1_20

M. van Sinderen et al. (Eds.): IWEI 2012, LNBIP 122, pp. 34 45, 2012.

(๑) IFIP International Federation for Information Processing 2012 
It is believed that the cost advantages of enterprise integration systems are significant [3]. The authors in [4] have estimated the benefits of improved information exchanges using electronic data interchange (EDI) between Chrysler and its suppliers to an annual amount of 220 million US\$. In another study in the health care industry, Walker et al. [5] assessed the net value of a fully implemented electronic health care information exchange and interoperability as $\$ 77.8$ billion per year. However, it should be considered that enterprise integration is expensive and time-consuming [3]. Gartner [6] estimates the total revenue of application integration infrastructure as $\$ 5$ billion per year in 2011.

The scientific literature has touched upon the topic of benefits and effects of enterprise integration on organizations from different viewpoints. However, there has been no effort to date to comprehensively evaluate these benefits. This paper outlines how enterprise integration can be useful to organizations, and provides guidelines for how future research might be conducted. In short, the purpose of this paper is to review the current state of research on the benefits of Enterprise Integration, thus providing a useful baseline for future research. This paper presents the literature review from a master thesis project [7] with the goal of developing a method capable of measuring the benefits of EI in quantitative terms.

\section{Defining Enterprise Integration}

A variety of terms are presented in the literature to denote the integration area. Examples of such terms are enterprise integration (EI) [8, 9], application integration (AI) [10], systems integration [11], and enterprise application integration (EAI) [3].

Vernadat [9] contrasts Enterprise Integration (EI) with systems interoperability, designating EI by a "strong organizational dimension" while considering interoperability more of a technical nature. From a pure technical perspective, EI is considered to denote connecting computer systems and IT applications to support business process operations [9]. On the other hand, from an organizational perspective, EI facilitates information, control, and material flows across organization by connecting all the necessary functions and functional entities to improve communication, cooperation, and coordination within the enterprise [9].

Adopting application integration (AI) term as the umbrella term for integration, the authors in [10] introduce three main subcategories, namely (1) intra-organizational AI, (2) inter-organizational AI, and (3) hybrid AI. They thus differentiate the integration of internal applications from cross-enterprise business processes and systems. The term hybrid is used to denote that in some cases, these applications function as intra-organizational AI and in others as inter-organizational applications.

Markus [11] defines systems integration as establishment of "tighter linkages between different computer-based information systems and databases". By this definition, the author views systems integration as a requirement to achieve business integration. In this context, enterprise application integration (EAI) is defined as an systems integration strategy to achieve data and process integration [11]. 
Lee et al. [3] uses enterprise integration and systems integration synonymously as "the capability to integrate a variety of different system functionalities". Similar to [11], Lee et al. [3] considers EAI as an approach to systems integration.

In this article enterprise integration (EI) is adopted to refer to the entire integration area. Most importantly, this definition of EI encompasses various perspectives and levels of integration, namely systems integration (interconnection of devices via computer networks), application integration (integration of software applications and database systems), and business integration (co-ordination of functions that manage, control and monitor business processes) [8]. Moreover, the term itself possess the required comprehensiveness to be considered as the umbrella term incorporating other related terms mentioned for integration.

\section{Benefits of Enterprise Integration}

The authors in [12] propose a framework as a decision-making tool regarding the adoption of EI. The proposed framework considers four integration layers, namely the connectivity layer, transportation layer, translation layer, and the process automation layer. In the connectivity layer, integration elements like data and objects are extracted from source systems and are transferred to the integration infrastructure by the transportation layer. Transported elements are translated and reformatted for target systems using the translation layer. The process automation layer is responsible for routing the translated elements to target systems.

Based on the classification of ERP benefits proposed by [13], Themistocleous [14] classifies the benefits of EI in five different types; organizational (e.g. more organized business processes), managerial (e.g. ROI), strategic (e.g. increase collaboration among partners), technical (e.g. achieve data, object and process integration), and operational (e.g. reduce cost). However, it could be argued that such scheme imposes a subjective classification rather than an objective one, i.e. the decision to classify one benefit in a category depends on the researcher rather than the characteristics of the benefit itself. In this sense, these types could overlap, which makes it is difficult in practice to make a distinction between them.

Enterprise Integration Council suggests flexibility and agility, defined as the ability to rapidly respond to new business opportunities, as the ultimate goals of EI [3]. Moreover, it proposes cycle time reductions, cost reductions, and cost containment as benefits of enterprise integration systems [3]. In acclaiming such benefits, Chari and Seshadri state that "adopting standards-based integration solutions is the most promising way to reduce the long-term costs of integration and facilitate a flexible infrastructure" [15].

Themistocleous and Irani [16] view the benefits of EI as reduced integration time, more flexible and maintainable solutions, and the easing of migration to new technologies due to conformance of EI systems to common standards [16]. They argue that these benefits ultimately result in reduction of overall integration costs due to the reduction of both integration time and maintenance costs. 
Ruh et al. [17] face the challenge of determining the effects of EI from the organizational perspective. The authors claim that an integrated infrastructure allows companies to improve their performance, increase their productivity and increase the quality of services offered to their customers. Similarly, Themistocleous and Irani assert that EI strengthens supply chains [16]. Manouvrier and Menard [1] also stress improvements in quality of service as the most significant benefit of integration systems.

From another point of view, Shin [18] claims that EI is generally effective for large firms, with size above some critical point. They stress that in order to rationalize the initial fixed cost of EI systems, companies shall have conditions such as a mass of complicated information systems.

In addition to increasing reactivity, adaptability and the ability to manage external exchanges optimally, Manouvrier and Menard [1] suggest accelerating time-to-market as another effect of EI.

Tackling the problem of determining the effects of EI, Themistocleous and Irani in [19] and [20] propose that Information systems that benefit from integration with others can arguably be viewed as no longer having a definitive start and end. The authors claim that such systems are evolving entities that grow and develop over time, in tune with the business environment.

Analyzing the benefits of enterprise integration on the data level, the authors in [21] suggest business benefits such as improved productivity, improved data accuracy, greater agility and flexibility, and system replacement/organizational mergers.

\subsection{A Classification of Benefits}

In order to present the identified benefits of EI for an enterprise, a classification scheme is employed. This scheme is intended to unite different perspectives of enterprise integration benefits that can be found in the literature. The classification scheme is based on the conceptual model proposed for enterprise integration in [22]. The authors in [22] proposed a model in which an enterprise is seen as a layered framework of related activities sharing common goals, which as a whole, describe an organization. Each of these layers can be seen independently as a view of the enterprise. These layers are network, information, application, work processes and organization. The integration goal at the network layer is to provide connectivity, defined as the linkages between systems, applications, and modules. Thus the integration issue addressed at this layer is the physical heterogeneity of the hardware and their operating systems in a physical network. In our model, however the notion of the network layer is excluded (cf. figure 1). Such exclusion could be justified considering that the network layer is deemed to be too fundamental to give rise to benefits.

The information layer is the view incorporating data sharing issues, as it involves enabling organizational sub-units to understand and use data from other subunits. The application layer describes the systems used by the organization. The integration goal at this layer is application interoperability which is defined as "the ability of one software application to access/use data generated by another software system” [22]. 
Tasks and the manner and order in which they are conducted in order to produce an output are the core of the work processes layer. In the last layer, organization layer is where the different strategies including business strategy, organizational design strategy and information systems strategy must all be aligned with one another.

The described scheme provides an abstraction mechanism in order to unite various perspectives of enterprise integration benefits in different literature. Moreover, it offers the possibility to picture the relation between various benefits related to the different layers.

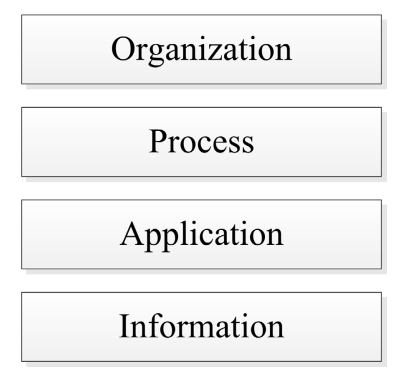

Fig. 1. Classification scheme; based on [22]

Table 1. Classified benefits of enterperise integration

\begin{tabular}{|l|l|}
\hline \multirow{5}{*}{ Organization } & - customer relationship improvements \\
& - enterprise flexibility improvements \\
& - costs reduction \\
& - revenue growth \\
& - supply chain improvements \\
\hline \multirow{4}{*}{ Process } & - Business-to-Business (B2B) processes improvement \\
& - decision making processes improvements \\
& - processes flexibility/agility improvements \\
\hline \multirow{4}{*}{ Application } & - processes performance improvements \\
& - applications switching costs decrease \\
& - data analysis capabilities improvements \\
& - total cost of ownership (TCO) decrease \\
& - systems reusability improvements \\
\hline Information & - data entry/processing automation and data quality improvements \\
& - enhanced data accessibility and reusability \\
& - superior data standardization \\
\hline
\end{tabular}


The presented scheme differs essentially from both models proposed in [12] and [14]. By classifying the workflow of a typical integration scenario, the model proposed in [12] provides a layered view over EI activities (how it is performed) rather than the benefits of EI (what is achieved). The scheme proposed in this paper, however, aims to describe an organization as a layered framework of related independent activities which share common goals. The classification presented in [14] provides a classification model for the benefits of EI. On the other hand, the model limits its perspective to the point of view of an organization's senior management.

With the aim of providing a comprehensive view over the benefits of EI, each of the layers in the presented scheme (cf. figure 1) can independently be seen as a view of the enterprise. In this way, the proposed scheme covers all the layers of an enterprise. This can also be justified by the similarity of the proposed layers to the enterprise layers in the enterprise architecture (EA) literature. For instance, the three layers presented in Archimate [23] could be associated with the proposed scheme:

- Archimate's technology layer could be associated with scheme's information layer;

- application layers of both could be related to each other;

- business layer of Archimate could be linked with the process and organization layers of the proposed scheme.

\subsection{Information Layer}

Benefits identified from the literature related to the information layer are data entry/processing automation and data quality improvements [14, 21], enhanced data accessibility and reusability $[3,9,14,17,21]$, and superior data standardization $[3,14]$.

Data Entry/Processing Automation and Data Quality. The authors in [21] discuss that enterprise integration minimizes data entry points, automates data entry, business rules execution against data, and data transformation. They argue that such automation minimizes the opportunity for human error, which in turn results in improvements in data accuracy. Moreover, according to [14], EI results in data with increased reliability, which is defined as a data quality dimension indicating whether the data can be relied on to convey the right information [24]. To indicate the significance of data quality improvements by enterprise integration, the authors in [14] discuss that such improvements supports the decision-making process, which in turn, improves performance and management of the organization.

Data Accessibility and Reusability. Providing support for more efficient data sharing and more reusable data is mentioned as one of the results of employing enterprise integration in organizations [14]. However, more emphasis has been placed on enhanced data accessibility, which is discussed to be achieved through information exchanges [17, 21]. More specifically, it is argued that enterprise integration improves data accessibility by facilitating greater degree of communication [3] by connecting all the necessary functions and heterogeneous functional entities [9]. 
Data Standardization. Considering automated data entry and execution of business rules against the data as a feature of enterprise integration, Lee et al. [3] argues that this would lead to improved data standardization. The authors in [14] posit that an implication of enterprise integration is reduced data redundancy, resulting in less data and application, and less maintenance effort.

\subsection{Application Layer}

The application layer contains the following benefits: applications switching costs decrease [3, 10], data analysis capabilities improvements [14], systems interoperability improvements [3, 9, 14, 25], systems modifiability improvements [1, 10, 14, 21, 25], total cost of ownership (TCO) decrease [10, 14, 26, 27], and systems reusability improvements [14, 27].

Applications Switching Costs. The authors in [10] and [3] argue that by enabling organizations to conform to common standards, enterprise integration facilitates easier and quicker migration to new technologies and reduces switching costs of systems.

Systems Interoperability. The literature relates improvements in systems interoperability achieved by enterprise integration to the standardization of interfaces [3, 14], which allows greater degree of cooperation between different applications [3, 9]. Brunnermeier et al. [25] discuss that enterprise integration reduces problems caused by imperfect interoperability.

Systems Modifiability. Providing a common integrated infrastructure, enterprise integration minimizes the changes to application code [10, 14], and leads to more flexible, manageable and maintainable solutions $[10,14,21]$ with higher scalability $[14,21]$. Moreover, mediation and transformation functionalities of enterprise integration and provided asynchronous communication results in higher degrees of loose coupling between applications [1], which yields in increased information systems flexibility and reactivity [1]. Additionally, enterprise integration makes it possible to position business rules outside the code for applications, thus rendering maintenance and adaptability of systems much simpler and faster. Furthermore, the authors in [25] discuss that the improvements in systems interoperability reduce related mitigation and reworking activities and corresponding time and resources, which could be discussed that increase systems modifiability.

Total Cost of Ownership (TCO). As discussed, lower applications switching costs and enhanced systems interoperability decrease the lifecycle cost of the information systems within the organization. Additionally, improved systems modifiability results in reduced purchasing, maintaining and training efforts [25]. In overall, enterprise integration reduces the total cost of ownership for information systems in the organization, including cost of managing, running and maintaining the IT infrastructure, and the time required to conclude systems integration $[10,14]$. On the other hand, increased flexibility and software reuse extends the information systems 
lifecycle, which could significantly reduce the total cost of ownership for information systems within the organization $[26,27]$.

Systems Reusability. The authors in [27] state that the new integrated information system resulting from integrating multiple information systems offers software reuse through the adaptability provided by integration. In the results of multiple case studies, Themistocleous [14] also supports this finding.

\subsection{Process Layer}

This layer includes Business-to-Business (B2B) processes improvement [1, 12, 14, 17], decision making processes improvements [14, 17], processes flexibility/agility improvements $[3,14,21,25]$, and processes performance improvements $[3,9,14,16$, $17,21,25]$.

Business-to-Business (B2B) Processes. The integrated IT-infrastructure enables sharing information between the partners of a supply chain [14, 17], which increases collaboration among trading partners [12], and strengthens supply chains [17]. Moreover, internal and external supply chains are integrated, resulting in improvements in supply chain planning. From the perspective of organization's customers, this would yield in improvements in the quality of services offered to customers [1].

Decision Making Processes. Enterprise integration mediates the flow of information from stovepipe applications to the common warehouse and supports the conversion of data from various applications' formats to a common format. This facilitates the construction of a data warehouse which could provide integrated information for decision support of the organization. [17]

Processes Flexibility/Agility. As a consequence of enterprise integration, process scalability is increased [14], delays in activities of value chain could be decreased [25], organization could quickly respond to changes in its environment by providing new products, services and information [3], quickly modifying its business methods [3], or bringing new business lines [21].

Processes Performance. The authors in [9] argue that by connecting all the necessary functions and heterogeneous functional entities (e.g. information systems, devices, applications, and people), enterprise integration facilitates information, control, and material flows across organization boundaries, aids data and information exchanges (i.e. interoperation between applications), which in turn improves timely orchestration of process steps within the enterprise. Moreover, business processes are optimized in terms of productivity by reducing manual tasks $[12,14]$, eliminating unnecessary or redundant tasks by letting systems share data across the enterprise [14, 21], providing employees with better access to the information they need to do their jobs [17], and reducing time and resource required for reworking tasks [25]. Additionally, if processes are reengineered during implementation of enterprise integration, business 
processes would become more organized, which would lead to increases in business processes understanding and control, resulting in increased performance [14].

\subsection{Organization Layer}

The layer with highest abstraction level encompasses the following benefits: customer relationship improvements [14, 17], enterprise flexibility improvements $[1,3,9,14$, $17,21]$, facilitating organizational mergers [21], costs reduction [14], revenue growth $[1,14,25]$, and supply chain improvements $[14,17,28]$.

Customer Relationship. In an integrated IT infrastructure, all information relevant to a customer would be available in an integrated form, which provides a 360-degree view of customer relationships, resulting in improved services quality for customers and increased services/products value to customers [14, 17]. Such increased value could build customer loyalty, which in turn could generate profit for the organization [29-31].

Enterprise Flexibility. Connecting all the necessary functions and heterogeneous functional entities allows for a greater degree of communication, coordination, and cooperation in human factors as well as information technologies. This enables organizations to continuously monitor market demand, quickly respond by providing new products, services, information, and application, and quickly introduce new technologies and modify business methods [3, 9, 21]. Moreover, not only does EI reduce the time-to-market $[1,14,17]$, but also due to cross-department standardization to all domains of the enterprise, the resulting increased reactivity can manifest itself by a reduction in loss of revenues or an increase of quality of service [1].

Organizational Mergers. In case of organizational mergers or acquisitions, when new business units enter the mix, enterprise integration facilitates incorporating new systems into the enterprise architecture [21].

Costs Reduction. Reengineering processes, during implementation of enterprise integration, results in more organized business processes and increases business processes understanding and control. This leads to business processes improvement, resulting in increased organizational performance.

Revenue Growth. Imperfect interoperability could lead to delays in key activities in value chain. Such delays yield to reduction in market share and the associated revenues. Another consequence could be delays in revenue [1, 14, 25].

Supply Chain. By integrating internal and external supply chains, enterprise integration improves planning in supply chain management [14], and therefore improves supply chains $[17,28]$.

\section{Discussion}

This research sought to reveal the current state of the scientific literature concerning the potential benefits of EI. Our research results provide a consolidated view of the scientifically established picture of such potential benefits. 
The literature suggests benefits of EI, related to various aspects of an enterprise. However, the results of many studies are limited to specific cases due to the research methodologies adopted. Some studies also suffer from limited understandability and reusability, since they lack a classification scheme or proper taxonomy. Moreover, disperse backgrounds of the studies conducted regarding EI benefits causes an ambiguity with regard to the context where the perceived benefits are applicable. To address such voids, the presented research provides an account of the available research findings of EI benefits, classified using a conceptual model of the enterprise.

The results have several implications for both researchers and practitioners. First of all, the provided information on potential EI benefits and their applicability (enterprise layer) enhances the understanding of EI. Moreover, the list and classification of EI benefits provided can function as a source for defining relevant objectives for EI projects, which is useful in business cases. Additionally, the results could be used to define EI effectiveness metrics. Within a more ambitious research agenda, the classified benefits could be employed to develop methods or models capable of measuring such benefits in financial terms.

Certain benefits have been found to semantically overlap or to group more than one notion, while some might appear vague or of high-level abstraction. This reflects the different intentions of the original studies' authors. However, the affected benefits were incorporated without any modifications to avoid potential semantic inconsistencies.

In addition to the context within which certain benefits appear as results of EI, future studies are recommended to understand and report the causal mechanisms behind these benefits. Identifying the relations between different benefits of EI with the specific mechanisms that generate them provides insight on how the benefits were actually brought about. Such causal relationships could offer additional and critical understanding regarding the benefits of EI, not least for decision-makers running actual integration projects.

Although the study aimed to be highly inclusive regarding the studies available on the subject of benefits of EI, it might not have covered the entire span of available literature. To increase the comprehensiveness, additional studies could be conducted to consider additional studies in other languages than English (which was the focus of the search in this study), as well as the gray literature (e.g. organizational statistics, internal reports).

Moreover, based on the results of this research, one future study could be to perform more rigorously designed EI research on benefits of EI. This would lead to more reproducible results, a crucial need for the area.

\section{References}

1. Manouvrier, B., Ménard, L.: Application Integration: EAI, B2B, BPM and SOA. WileyIEEE Press (2008)

2. Fulk, J., DeSanctis, G.: Electronic communication and changing organizational forms. Organization Science 6, 337-349 (1995) 
3. Lee, J., Siau, K., Hong, S.: Enterprise integration with ERP and EAI. Commun. ACM. 46, 54-60 (2003)

4. Mukhopadhyay, T., Kekre, S., Kalathur, S.: Business Value of Information Technology: A Study of Electronic Data Interchange. MIS Quarterly 19, 137-156 (1995)

5. Walker, J., Pan, E., Johnston, D., Adler-Milstein, J., Bates, D.W., Middleton, B.: The value of health care information exchange and interoperability. Health Affairs, W5:10-W5:18 (2005)

6. Lheureux, B.J., Skybakmoen, T., Thompson, J., Malinverno, P., Biscotti, F., Wilson, D.R., Schulte, W.R., Beyer, M.A., Knipp, E., Pezzini, M.: Taxonomy, Definitions and the Vendor Landscape for Application Integration Solutions (2011)

7. Fazlollahi, A.: Benefits of Enterprise Integration Systems (Master thesis). KTH, Industrial Information and Control Systems, Stockholm (2012)

8. Chen, D., Doumeingts, G., Vernadat, F.: Architectures for enterprise integration and interoperability: Past, present and future. Computers in Industry 59, 647-659 (2008)

9. Vernadat, F.B.: Enterprise Integration and Interoperability. In: Nof, S.Y. (ed.) Springer Handbook of Automation, pp. 1529-1538. Springer, Heidelberg (2009)

10. Themistocleous, M., Irani, Z.: Novel taxonomy for application integration. Benchmarking: An International Journal 9, 154-165 (2002)

11. Markus, M.L.: Paradigm shifts-E-business and business/systems integration. Communications of the Association for Information Systems 4 (2000)

12. Themistocleous, M., Irani, Z.: Towards a novel framework for the assessment of enterprise application integration packages. In: Proceedings of the 36th Annual Hawaii International Conference on System Sciences. IEEE (2003)

13. Shang, S., Seddon, P.B.: A comprehensive framework for classifying the benefits of ERP systems. In: AMCIS 2000 Proceedings, p. 39 (2000)

14. Themistocleous, M.: Justifying the decisions for EAI implementations: a validated proposition of influential factors. Journal of Enterprise Information Management 17, 85-104 (2004)

15. Chari, K., Seshadri, S.: Demystifying integration. Communications of the ACM 47, 58-63 (2004)

16. Themistocleous, M., Irani, Z.: Benchmarking the benefits and barriers of application integration. Benchmarking: An International Journal 8, 317-331 (2001)

17. Ruh, W.A., Brown, W.J., Maginnis, F.X.: Enterprise application integration: a Wiley tech brief. John Wiley \& Sons, Inc., New York (2001)

18. Shin, I.: Adoption of Enterprise Application Software and Firm Performance. Small Business Economics 26, 241-256 (2006)

19. Puschmann, T., Alt, R.: Enterprise application integration-the case of the Robert Bosch Group. IEEE Comput. Soc. (2001)

20. Themistocleous, M., Irani, Z., Psannis, K., Vrehopoulos, A.: Application Integration of Information Technology: Classification of Benefits and Barriers. Information Systems, 156-164 (2001)

21. Gleghorn, R.: Enterprise application integration: a manager's perspective. IT Professional, 17-23 (2005)

22. Giachetti, R., Hernandez, P., Nunez, A., Truex, D.: A research framework for operationalizing measures of enterprise integration. Knowledge Sharing in the Integrated Enterprise, 237-247 (2005)

23. The Open Group: ArchiMate ${ }^{\circledR} 2.0$ Specification. The Open Group, Berkshire, UK (2012)

24. Wand, Y., Wang, R.Y.: Anchoring data quality dimensions in ontological foundations. Communications of the ACM 39, 86-95 (1996) 
25. Brunnermeier, S.B., Martin, S.A.: Interoperability costs in the US automotive supply chain. Supply Chain Management: An International Journal 7, 71-82 (2002)

26. Themistocleous, M., Irani, Z., Kuljis, J., Love, P.E.D.: Extending the information system lifecycle through enterprise application integration: a case study experience. In: Proceedings of the 37th Annual Hawaii International Conference on System Sciences, p. 8 (2004)

27. Irani, Z., Themistocleous, M., Love, P.E.D.: The impact of enterprise application integration on information system lifecycles. Information \& Management 41, 177-187 (2003)

28. Mentzer, J.T., DeWitt, W., Keebler, J.S., Min, S., Nix, N.W., Smith, C.D., Zacharia, Z.G.: Defining supply chain management. Journal of Business Logistics 22, 1-26 (2001)

29. Hallowell, R.: The relationships of customer satisfaction, customer loyalty, and profitability: an empirical study. International Journal of Service Industry Management 7, 27-42 (1996)

30. Edvardsson, B., Johnson, M., Gustafsson, A., Strandvik, T.: The effects of satisfaction and loyalty on profits and growth: Products versus services. Total Quality Management \& Business Excellence 11, 917-927 (2000)

31. Heskett, J.L., Jones, T.O., Loveman, G.W., Earl Sasser Jr., W., Schlesinger, L.A.: Putting the service-profit chain to work. Harvard Business Review 72, 164-174 (1994) 